\title{
The Perioperative Nursing of Treating the Elderly Patients of Femoral Intertrochanteric Fracture with PFNA
}

\author{
Ying Zhang* \\ Traditional Chinese Medicine Hospital of Daozhen Autonomous County, Zunyi, Guizhou 563000, China
}

\begin{abstract}
Objective: Summarize the perioperative nursing of the elderly patient of femoral intertrochanteric fracture. Method: Retrospectively analyze the preoperative preparations as well as the postoperative nursing of treating the 75 elderly patients of femoral intertrochanteric fracture with PFNA. Results: Those 75 patients all pull through perioperative period and their functions are in good recovery. Conclusion: The elderly patient of femoral intertrochanteric fracture has much surgical risk together with many complications during perioperative period. Adopting effective comprehensive nursing can improve the treatment effect of the operation, and can also reduce the complications.
\end{abstract}

\section{KEYWORDS}

Elderly patients

Intertrochanteric fracture

Perioperative nursing

\section{Introduction}

The study of the epidemiology shows that with the obvious increase of life expectancy, the incidence of hip fracture increases constantly. The femoral intertrochanteric fracture is especially common among the elderly. There exists osteoporosis among them, and their bones are becoming weakened, so most of them are easy to fracture bones when they suffer a low or moderate trauma, such as falling down. Moreover, the femoral intertrochanteric fracture is always found among the old people. Nowadays, operative treatment, early functional exercise and early weight bearing exercise are proposed during the clinical treatment to reduce the incidence of the complications. At present, PFNA is a common internal fixative method to treat the elderly patients of femoral intertrochanteric fracture. It has lots of advantages [1], such as credible fixation, simplicity of operation, low harm, convenience for the early ambulation and high rate of fracture healing. We perform minimally invasive PFNA operation, and strengthen periopera-

Copyright @ 2015 Ying Zhang

doi: 10.18686/jn.v4i1.1

Received: November 9, 2014; Accepted: January 18, 2015; Published online: March 26, 2015

This is an open-access article distributed under the terms of the Creative Commons Attribution Unported License (http://creativecommons.org/ licenses/by-nc/4.0/), which permits unrestricted use, distribution, and reproduction in any medium, provided the original work is properly cited.

${ }^{\star}$ Corresponding author: Traditional Chinese Medicine Hospital of Daozhen Autonomous County, Zunyi, Guizhou 563000, China. E-mail: 631753846@qq.com tive nursing at the same time. Reviewing the 75 patients of femoral intertrochanteric fracture who received treatment in the Orthopedics Department in our hospital from the February in 2014 to the same month in 2015, we can find that we have gained delightful effect through scientific and effective operative treatments as well as the nursing and rehabilitation. Now it is reported as follows.

\section{Clinical information}

\subsection{General information}

There are 75 patients in the group, among which there are 20 males and 55 females; their ages are from 63 to 93, and the average age is 75 years old. There are obvious shortening and external deformity of the wounded limb on the left parts of 36 patients, while on the right parts of 39 patients, and their activities are all restricted obviously. Moreover, before the fracture, there are 46 patients with hypertension, 35 patients with diabetes, and 11 patients with hemiplegia.

\subsection{Fracture classification and therapeutic method}

According to the AO Classification [2]: Type I: 11 cases, Type II: 60 cases, Type III: 4 cases. Therapeutic method: The elderly patients of femoral intertrochanteric fracture who received treatment in our Orthopedics Department are all conventionally treated with the internal fixative method-PFNA.

\subsection{Results}

The lengths of stay are from 6 to 18 days, and the average 
length is 9 days. There is no patient who catches serious complications, such as bedsores, deep venous thrombosis, urinary system infection, pulmonary infection and heartlung failure. All the patients pull through perioperative period, and their functions are in good recovery. Those 75 patients in the group all get the follow-up visit with time of 6 to 16 months, and the average time is 12 months. According to the functions of Hip Joint Harris [3] to give the finial marks, among which there are 70 cases $(93.3 \%)$ are excellent and 5 cases (6.7\%) are good.

\section{Perioperative nursing \\ 3.1. Preoperative nursing \\ 3.1.1. Mental nursing}

The elderly patients of femoral intertrochanteric fracture need to stay in bed for a long time, which will bring great inconvenience to their lives, at the same time they also need to bear physical pain as well as the worry about the operation result. All of these will add their pressure mentally, thus they may have psychological disorder caused by their nervous, fear, worry and restlessness. Moreover, they will even have resentment against the operative treatment. Therefore, it is important to understand and master patients' mental activities in detail, give them necessary consolation, explanation and encouragement, and explain the operative method (it is better to explain coordinating with the doctor's operating video), objective and clinic effect of the PFNA internal fixation patiently. Meanwhile, we had better invite the patients who are postoperative to show up to talk with them, so that patients' psychological disorder can be reduced. Then they can accept the operation and nursing in good mentality [4].

\subsubsection{Preoperative preparations}

Because of the modern anesthetic medicine and the development of technology, the operative methods become more appropriate and more reasonable. And with the popularization of the monitor and test methods during the perioperative period, we have a better understanding about the complications (even the deadly ones) that brought by long-time stay in bed during the process of non-operative treatment. Moreover, the idea that cures the hip fracture of the elderly by operation is accepted widely. It is important to do the case observation, thus the preoperative examination should be completed as soon as possible, including complete cardiovascular ultrasonic examination, arteriovein ultrasonic examination of the lower limb, and pulmonary ventilation function examination. We should arrange the operation for the patient who cannot tolerate the surgery as soon as possible [5].

\subsubsection{The nursing for the skeletal traction before op- eration}

We should do the skeletal traction for the patients of femoral intertrochanteric fracture before operation gener- ally, and the traction weight is about $1 / 10$ to $1 / 7$ of body weight. During the process of traction reduction of the lower limbs, because the most pressure is concentrated on the sacrum and foot, and the traction is a braking process, it is easy to form pressure sores on the parts mentioned above. Thus the skin should be kept dry, and we should avoid damaging skin by dragging or rubbing when setting the body position. In addition, cushion should be put appropriately under the bone apophysis parts mentioned above, so that the bruise can be prevented during the traction. We need regularly observe whether the traction line and the thigh are on the same horizontal axis, whether the traction hammer is drop, and whether feet touch the footboard of the bed. Moreover, the traction should be adjusted regularly to assure that it is effective. The mouth of the nail road should be disinfected 2 times a day by the iodophor solution to prevent the nail road from being infected. During the traction, we should pay attention to the blood circulation and neurological function of the limbs, avoiding forming the deep venous thrombosis or over traction in one's nerve.

\subsection{Intraoperative nursing}

During the operation, the room temperature should be controlled at $22-24{ }^{\circ} \mathrm{C}$ and the humidity should be kept in $50-60 \%$. Before the operation, we should check carefully the patient's name, name of the operation, surgery spot and conditions of preoperative preparations; check whether the patient wear false-teeth and metal objects; set a comfortable body position for the patient; open the vein tunnel after the patient entering the room. After helping anesthetist to do the anesthesia work, one is technically responsible for the traction and fixation of the wounded limbs, and places the brace on the perineum part to resist the traction. Then we should place the hand bracket with our multiple upper limbs' abduction smaller than 70 degrees, so that it is convenient for us to observe during the operation. Watch the patient closely, pay attention to his consciousness, breath and the changes of his vital signs, and strengthen the inspection. Send the patient back to the ward together with anesthetist after the operation, and appropriately place and fix all kinds of drainage tubes.

\subsection{Postoperative nursing \\ 3.3.1. Postoperative base nursing care}

These groups of patients are dealt with general anesthesia or spinal epidural anesthesia mode to return the wards after awakened. The first level of nursing after the surgery, ECG monitoring, oxygen flow $3 \mathrm{~L} / \mathrm{min}$, supervision of $\mathrm{BP}$, $\mathrm{R}, \mathrm{P}, \mathrm{SpO}_{2}$, and the awareness and consciousness to the stable vital signs. Assist the patient to sleep flatly without pillow, and keep the affected limb in neutral position with the outreach of $15^{\circ}$ to avoid adduction, internal rotation and external rotation with the lower limbs padded about 10 $\mathrm{cm}$ high to benefit the venous return and reduce swelling. 
After no eating and drink for $6 \mathrm{~h}$ begin with the semi-flow diet, and avoid foods of spicy which stimulating and easy to cause abdominal distension, and recovery the same preoperative diet after gastrointestinal peristalsis. It is allowed to increase the food rich in crude fiber and calcium and massage the abdomen in a clockwise direction to promote intestinal peristalsis and prevent constipation. Generally catheterizations are reserved after operations, for monitoring $24 \mathrm{~h}$ urine volume and color, and if the abnormal appears, there will be timely symptomatic treatment, and as early as possible remove the catheter [6].

\subsubsection{Complication prevention and nursing cares}

Because this group of patients are elderly people, and most have not completed heart and lung function and many basic diseases are more, so it is necessary to carry out prevention and nursing cares for all kinds of complications. Assisting patients to turn over, keep the skin clean and dry, and massage the pressed on the skin, and once every 2 hours, place a soft pillow in the hip and ankle bone and other prominent part, and if necessary it is allowed with air cushion bed to prevent pressure sores. Guide and encourage patients with deep breathing and effective cough, expectoration, and give appropriate sit-back slapping and give aerosol inhalations twice daily to prevent pulmonary infection. Encourage patients to drink with more water, and give perineal scrubs twice daily, and every 2-4 hours open the catheter for the one with urinary catheter reserved for exercising voiding functions and the early removal of catheters and the prevention of urinary system infection. The occurrence rate of deep venous thrombosis (DVT) after orthopedic operations has an increasing trend [7], and aged patients are groups with high risks of thrombosis. The majority of DVT occur during the surgery and in $4 \mathrm{~d}$ after surgery.

\subsubsection{Functional exercise}

Guide the patient to do dordoflexion and flexor digitorium of the afftected limb, 3-5 minutes once, and 4-6 times a day. Instruct the patient to do and diastolic exercise of musculi quadriceps femoris under static condition: Stretch the leg straight with the heel backward, 15-20 minutes once, and 4-5 groups once. On the second day of postoperation, guide the patients to do contraction and diastolic exercise of musculi quadriceps femoris; Instruct the patient to stretch his knee joints straight, dorsally extend his ankle joints, in the meantime, his knee joints should be pressed on the bed surface, these should last for 5-10 seconds before relaxation, 3-4 times a day, 50 times once. On third day of post-operation, guide the patient to tighten the muscle of his affected limb for 9 seconds, and then relax for 3 seconds, 30 minutes once and 3-4 times a day. In a meantime, the patient should do passive functional exercise of his affected limb cooperating with the CPM machine, beginning from $30^{\circ}$ and this should be gradually increased but not exceed $90^{\circ}$. In the sixth week of postoperation, when callus is in the mature period, the patient can be guided to do weight-bearing exercise gradually. Transit from partly weight-bearing with a stick to weightbearing walking, until the single affected leg is able to bear his whole body weight. In third month, the stick can be abandoned according to condition.

\subsubsection{Guidance of hospital discharge}

Inform to dismantle stitches 10-12 d after surgery. Work out the specific training programs, and explain the importance of limb functional exercise so that patients and their families can master the training methods and precautions, and maintain a gradual functional exercise, prevent the fall, avoid failure of internal fixation and the other fractures. Note to control the primary disease, take the nutritious and digestible foods with protein, vitamins, and calcium, utilize the anti-osteoporosis drugs rationally, and promote the bone healing. Return visit at outpatient monthly for 3 months after surgery, in principle, check in month 6, 9, 12, adjust according to fracture healing, and visit for any discomfort [8].

\section{Conclusion}

As the aging of social population, the intertrochanteric fracture of elderly people trends to go up significantly in China. The perioperative nursing to elderly people has a certain particularity and challenging due to the degradation of their own organ function accompanied by one more of the underlying disease usually. Intertrochanteric fracture is a common disease in the elderly population associated with the severe osteoporosis and medical diseases usually. There are several common surgical treatments, it is not necessary to peel the soft tissue of fracture due to the surgical treatment of intramedullary fixation, which can reduce surgical trauma, complications, obtain a good fixation, benefit for the early functional exercise and have the other advantages, so it tends to the surgical treatment of intramedullary fixation currently. Therefore, the treatment of intertrochanteric fracture surgery lies not only on the superb technology, but also requires comprehensive, rational and effective nursing intervention for the success of the operation. Based on the 75 cases of intertrochanteric fractures, PFNA surgery perioperative nursing, the authors thinks that the good psychological intervention, the careful preoperative health assessment and preparation and the advanced painless care after surgery with the positive scientific and effective rehabilitation exercises can maximize to reduce the burden on the patient, reduce the pain of patients, reduce the incidence of various complications, promote early recovery of patients and improve their quality of life.

\section{References}

1. Thomas P, Ruedi, Richard EB, Christopher GM. Principle of fracture healing (2nd expanded edition). Translated 
by Shanghai: Shanghai Science and Technology Press. 2010:561.

2. Zhixiong L. Evaluation standard of orthopedics commonly used diagnostic classification method and function result. Beijing: Beijing Science and Technology Press. 2005:268270.

3. Yan LH, Zhang Q. Nursing of elderly intertrochanteric fracture fixation PFNA. Basic Medical Forum. 2013;17(9):1121-1122.

4. Huanjin $\mathrm{H}$. The nursing of perioperative period of curing intertrochanteric fracture in aged patients with PFNA. Qiqihar Medical University Journal. 2012;33(22):31433144.
5. Mingzhu Z, Jingui $X$, Jiacheng $H$. Nursing of elderly intertrochanteric fracture treatment with rotary prevention proximal femoral nail. Massage and Rehabilitation Medicine. 2012;39(20):124-125.

6. Liu H. Perioperative nursing of elderly intertrochanteric fracture. Massage and Rehabilitation Medicine. 2012;03(8):131-132.

7. Yang G. Perioperative nursing of intertrochanteric fractures pfna surgery. Journal of Chinese People's Armed Police Force Academy. 2013;15(4):326-327.

8. Yang L. Perioperative nursing of elderly rotary femur with pfna internal fixation. Basic Medical Forum. 2012;16(33):4404. 